\title{
MicroRNA-383 upregulation protects against propofol-induced hippocampal neuron apoptosis and cognitive impairment
}

\author{
XINLEI WANG ${ }^{1}$, GUOYOU DING ${ }^{2}$, WEI LAI ${ }^{2}$, SHIWEN LIU ${ }^{3}$ and JUN SHUAI ${ }^{2}$ \\ ${ }^{1}$ Department of Anesthesia, The First Affiliated Hospital of Nanchang University, Nanchang, Jiangxi 330006; \\ ${ }^{2}$ Department of Anesthesia, Chinese People's Liberation Army No. 94 Hospital, Nanchang, Jiangxi 330002; \\ ${ }^{3}$ Department of Anesthesia, Ganzhou People's Hospital, Ganzhou, Jiangxi 310000, P.R. China
}

Received August 18, 2016; Accepted May 11, 2017

DOI: $10.3892 /$ etm.2018.5838

\begin{abstract}
Anesthesia-induced cognitive impairment is a recognized clinical phenomenon. The present study aimed to investigate the effect of microRNA-383 (miR-383) expression on propofol-induced learning and memory impairment. In total, 48 male Sprague-Dawley rats (weight, $250 \pm 10 \mathrm{~g}$ ) were randomly divided into four groups ( $\mathrm{n}=12$ each): Control group, and three groups of rats that were anesthetized with propofol for $6 \mathrm{~h}$ and untreated (propofol model group), treated with a constructed lentivirus vector expressing miR-383 mimics (mimic + propofol group), or treated with miR-383 scramble (scramble + propofol group). The learning memory ability, hippocampal neuron apoptosis and expression of apoptosis-associated factors were detected using reverse transcription-quantitiative polymerase chain reaction and western blot analysis. Propofol treatment significantly reduced the relative mRNA and protein expression of miR-383, induced neuron apoptosis, upregulated the $\mathrm{Bax} / \mathrm{Bcl}-2$ ratio, downregulated the relative mRNA and protein expression levels of postsynaptic density protein 95 and cAMP-response element binding protein, and inactivated the phosphoinositide 3-kinase/protein kinase B signaling pathway. By contrast, miR-383 mimics significantly altered the propofol-induced dysregulation of the aforementioned factors. In conclusion, miR-383 mimic was able to repair propofol-induced cognitive impairment via protecting against hippocampal neuron apoptosis and dysregulation of related factors. The present study suggested that miR-383 may be used as a potential therapeutic target for the clinical treatment of cognitive impairment induced by propofol anesthesia.
\end{abstract}

Correspondence to: Dr Guoyou Ding, Department of Anesthesia, Chinese People's Liberation Army No. 94 Hospital, 1028 Jinggangshan Avenue, Nanchang, Jiangxi 330002, P.R. China E-mail: guoyouding77@163.com

Key words: microRNA-383, propofol, neuron apoptosis, learn and memory

\section{Introduction}

Cognitive dysfunction or impairment is prevalent in older adults with Alzheimer's disease $(1,2)$, or older individuals receiving anesthesia and surgery $(3,4)$. It is reported that $10-60 \%$ of elderly surgical patients have suffered with postoperative delirium and cognitive dysfunction in various surgical procedures (5). However, there is still no effective treatment to prevent or alleviate this complication. Similar to other diseases, cognitive impairments are caused by the abnormal accumulation of genetic or non-genetic alterations, and the therapy of cognitive impairments in older adults is a long and arduous process with limited apparent results (6). Anesthesia-induced cognitive impairments are associated with dysregulation of gene expression and neuron cell apoptosis. For instance, inactivation of the phosphoinositide 3-kinase/protein kinase $\mathrm{B}$ (PI3K/Akt) signaling pathway significantly increases the ratio of $\mathrm{B}$-cell lymphoma-2-associated $\mathrm{X}$ protein $(\mathrm{Bax}) / \mathrm{B}$-cell lymphoma-2 (Bcl-2) resulting in increased hippocampal apoptosis (7). Certain microRNAs (miRs) are also involved in this procedure. For example, miR-132 mediates stress-inducible cognitive deficits via acetylcholinesterase (8), and miR-137 variants serve as negative predictors of impaired cognitive subtype of schizophrenia (9). Cell apoptosis is a complicated process involving various genetic factors, including brain-derived neurotrophic factor (10), postsynaptic density protein 95 (PSD95) (11), cAMP-response element binding protein (CREB) $(12,13)$, miR-34a (14), miR-383 (15), and the PI3K/Akt signaling pathway $(16,17)$. The PI3K/Akt signaling pathway is essential for cell apoptosis, and its inhibition has been proven to contribute to cancer cell apoptosis (16-18). This pathway is associated with cognitive impairments induced by diabetes, neonatal hypoxic ischemic brain damage, cardiovascular disease and anesthesia (19-21). Furthermore, miR-383 has been previously identified as an underexpressed miRNA in medulloblastoma $(22,23)$, and its downregulation promoted cancer cell proliferation and invasion (24). The in vitro effects of miR-383 appear to be partially dependent on p21 ${ }^{\text {Cip1 }}$ (23), which is a cell cycle inhibitor that serves a crucial role in several biological processes, including cell cycle and apoptosis (25). Additionally, Chakraborty et al (26) have demonstrated that miR-383 was associated with PI3K/Akt signaling pathway. However, to the best of our knowledge, no studies have 
examined the influence of propofol anesthesia-induced cognitive impairment on miR-383 expression, or focused on the association between propofol anesthesia-associated miR-383 expression and the PI3K/Akt signaling pathway.

In the present study, with the aim to investigate the effect of miR-383 expression on propofol-induced learning and memory impairment, a cognitive impairment rat model was established using propofol anesthesia. The effect of miR-383 expression on propofol anesthesia-induced cognitive impairment was analyzed using constructed lentivirus vectors expressing miR-383 mimics. Cell apoptosis, rat learning and memory abilities, and the expression of genetic factors were also examined. The current study attempted to provide information on the association between anesthesia-induced cognitive impairment and miR-383 expression, and assist in the development of a therapeutic strategy focusing on miR-383 for cognitive impairment.

\section{Materials and methods}

Animal anesthesia model. A total of 48 male Sprague-Dawley rats (7-week-old; weight, $250 \pm 10 \mathrm{~g}$ ) were obtained from Vital River Laboratory Animal Technology Co., Ltd. (Beijing, China). Prior to experiments, animals were housed at $22^{\circ} \mathrm{C}$ and $50 \%$ humidity under a controlled 12-h light-dark cycle with ad libitum access to food and water for 1 week for acclimatization. Rats were then randomly divided into four groups ( $\mathrm{n}=12$ each): Control group, and three propofol-anesthetized groups that were untreated (propofol group), treated with miR-383 mimics (mimic + propofol), and treated with miR-383 scramble (scramble + propofol). Animals in the three propofol groups were anesthetized for $6 \mathrm{~h}$, between 9:00 a.m. and 3:00 p.m., by intraperitoneal injection of $300 \mathrm{mg} / \mathrm{kg}$ body weight propofol $(833 \mu \mathrm{g} / \mathrm{kg} / \mathrm{min}$; AstraZeneca plc, London, UK) for 7 days. The control rats were anesthetized with the same conditions $\left(37^{\circ} \mathrm{C}\right)$, administrated with normal saline at $1 \mathrm{ml} / \mathrm{h}$ between 9:00 a.m. and 3:00 p.m., and allowed to breath regular air for $6 \mathrm{~h}$. Animals were allowed to recover for 7 days after anesthesia, and were then subjected to further tests or sacrifice. All protocols of animal experiments were reviewed and approved by the Institutional Animal Care and Use Committee at the Chinese People's Liberation Army No. 94 Hospital (Nanchang, China).

In vivo hippocampal injection of miR-383 lentivirus. For the induction of hippocampal miR-383 expression, the lentiviral construct was used. Briefly, the purchased coding oligonucleotides of antisense mouse miR-383 mimics and scramble sequence (Guangzhou RiboBio Co., Ltd., Guangzhou, China) were cloned and inserted into a lentivirus expression vector, Pcdh-CMV-MCS-EF1-copGFP (System Biosciences, Inc., Morrisville, PA, USA). The miR-383 mimics and scramble viral particles were produced in 293T cells (CRL-3216; ATCC, Manassas, VA, USA) via lentivirus expression vector co-expressed with pPACK packaging system (Systems Biosciences, Palo Alto, CA, USA). For the miR-383 transfection, rats were administrated with lentiviruses containing miR-383 mimics or scramble at $24 \mathrm{~h}$ before the first administration of propofol. A total volume of $2 \mu \mathrm{l}$ lentivirus-containing mimics or scramble vectors were injected to the hippocampus of the rats through a drilled hole $(0.05 \mathrm{~mm}$ in diameter $)$ on the right cortex just above the dorsal hippocampus, via a Hamilton syringe under a surgical microscope.

Morris water maze (MWM) test. Subsequent to treatment and propofol anesthesia, the MWM test was used to examine the spatial learning and memory of rats. At 7 days after anesthesia (recovery), half of the animals in each group were subjected to the test. The MWM, placed in a room with dimmed lights, was composed of a circular water pool (colored with black ink; $100 \mathrm{~cm}$ in diameter, $50 \mathrm{~cm}$ in height) with $30-\mathrm{cm}$ depth of water and a platform at $1 \mathrm{~cm}$ below the water surface $(12 \mathrm{~cm}$ in diameter). The maze was divided into four quadrants. Animals attempted to find the platform by swimming in each of the four quadrants every day for 5 consecutive days. After 5 days of training, swimming data was recorded on day 6 . At the beginning of the trail, animals were immersed in the center of one of the three quadrants without the hidden platform. A maximum of $60 \mathrm{sec}$ was allowed for finding the hidden platform, and at the end of each swim, rats were allowed to rest for $30 \mathrm{~min}$. A video camera on the ceiling was used to videotape the performance of the rats in association with their swimming ability. The time taken to reach the hidden platform (latency; recorded in sec), path length (cm) and swimming speed $(\mathrm{cm} / \mathrm{s})$ were analyzed using image tracking software (HVS Image 2020 Plus tracking system; HVS Image Software Ltd., Buckingham, UK). The latency was positively correlated with the rats' spatial memory.

Terminal deoxynucleotidyl transferase dUTP nick end labeling (TUNEL) assay. The apoptosis of hippocampal neurons was detected using TUNEL assay. Briefly, at 7 days after anesthesia, rats were sacrificed and $4-\mu \mathrm{m}$ paraffin-embedded hippocampal sections were prepared and de-paraffinized. A colorimetric TUNEL kit (EMD Millipore, Billerica, MA, USA) was then used for in situ apoptosis detection of apoptotic hippocampal neurons following the manufacturer's instructions. The apoptotic hippocampal neurons were stained a brown coloration when reacted with DAB. Cells in five arbitrarily selected fields were counted, and the percentage of TUNEL positive cell number was calculated.

Reverse transcription-quantitative polymerase chain reaction $(R T-q P C R)$. For RT-qPCR analysis, total RNA was extracted from hippocampus tissues 7 days after anesthesia using TRIzol reagent (Invitrogen; Thermo Fisher Scientific, Inc., Waltham, MA, USA). Then, $2 \mu \mathrm{g}$ of total RNA was used as a template to synthesize the first-strand cDNA using iScriptTM Reverse Transcription Supermix (Bio-Rad Laboratories, Inc., Hercules, CA, USA), and the mRNA and miRNA expression levels were determined using a Ssofast EvaGreen Supermix kit (Bio-Rad Laboratories, Inc.) with an ABI 7500 Fast Real-Time PCR system (Applied Biosystems; Thermo Fisher Scientific, Inc.) and a miRCURY LNA microRNA Array kit (version 14.0; Exiqon; Qiagen, Hilden, Germany). The primers (Table I) were synthesized by Shanghai Sangon Biologic Engineering Technology and Services Co., Ltd. (Shanghai, China). GAPDH was used as the internal control for mRNA quantifications and U6 was used as the internal control for miRNA quantification. 
Table I. Primers used for reverse transcription-quantitiative polymerase chain reaction.

\begin{tabular}{|c|c|}
\hline Gene & Primer sequence \\
\hline \multirow[t]{2}{*}{ miR-383 } & Forward, 5'-CTTTCCCAAGAGTTTCACT-3' \\
\hline & Reverse, 5'-CCACTCCAGTCCACCAAAT-3' \\
\hline \multirow[t]{2}{*}{ U6 } & Forward, 5'-CGCTTCGGCAGCACATATAC-3' \\
\hline & Reverse, 5'-TTCACGAATTTGCGTGTCAT-3' \\
\hline \multirow[t]{2}{*}{ Bax } & Forward, 5'-TTTGCTTCAGGGTTTCATCC-3' \\
\hline & Reverse, 5'-ATCCTCTGCAGCTCCATGTT-3' \\
\hline \multirow[t]{2}{*}{ Bcl-2 } & Forward, 5'-ATTGTGATGGTCATATTATTG-3' \\
\hline & Reverse, 5'-ATATCATATGTCCTTTATGCA-3' \\
\hline \multirow[t]{2}{*}{ GAPDH } & Forward, 5'-GAAGGTGAAGGTCGGAGTCA-3' \\
\hline & Reverse, 5'-TTGAGGTCAATGAAGGGGTC-3' \\
\hline
\end{tabular}

miR, microRNA; Bcl-2, B-cell lymphoma-2; Bax, Bcl-2-associated X protein.

The reaction conditions were as follows: $95^{\circ} \mathrm{C} 10 \mathrm{~min}$, followed by $95^{\circ} \mathrm{C}$ for $30 \mathrm{sec}, 60^{\circ} \mathrm{C}$ for $40 \mathrm{sec}$ and $72^{\circ} \mathrm{C}$ for $10 \mathrm{sec}$ for 40 cycles. All reactions were run in triplicate. The relative mRNA expression level was calculated by the $2^{-\Delta \Delta \mathrm{Cq}}$ method (27).

Western blot analysis. Animals were sacrificed 7 days after anesthesia, and the hippocampus tissues were extracted using lysis buffer [300 mM NaCl, $50 \mathrm{mM}$ Tris- $\mathrm{HCl}$ ( $\mathrm{pH} 7.5)$, 5 mM EDTA, 0.1\% NP-40, 1 mM DTT, $1 \mathrm{mM}$ phenylmethanesulphonyl fluoride, and 1:100 protease inhibitor cocktail], homogenized and centrifuged at $4^{\circ} \mathrm{C}$ at $12,000 \mathrm{x} \mathrm{g}$ for $10 \mathrm{~min}$ to collect the supernatants. Concentration of protein was determined using the bicinchoninic acid method. Hippocampus proteins were mixed with and boiled in sodium dodecyl sulfate (SDS) sample buffer for $10 \mathrm{~min}$, then $10 \mu \mathrm{g}$ protein was prepared for western blot analysis. Next, $10 \%$ SDS-PAGE was used to separate proteins, which were then electrophoretically transferred to polyvinylidene difluoride membranes (Invitrogen; Thermo Fisher Scientific, Inc.). Subsequent to blocking with bovine serum albumin (Sigma-Aldrich; Merck KGaA, Darmstadt, Germany) at room temperature for $1 \mathrm{~h}$, membranes were incubated at $4^{\circ} \mathrm{C}$ overnight with primary antibodies against the following: Bcl-2 (1:5,000, catalog no. 4223), Bax (1:1,000, catalog no. 610982), PSD95 (1:2,000, catalog no. 610495), phosphorylated (p)-CREB (1:1,000, catalog no. 7722), CREB (1:1,000, catalog no. 9197), p-PI3K (1:1,000, catalog no. 4228), PI3K (1:2,000, catalog no. 9655), p-Akt (1:1,000, catalog no. 4060), Akt (1:1,000, catalog no. 4691) and GAPDH (1:1,000, catalog no. 560792). The anti-BCL-2, p-CREB, CREB, p-PI3K, PI3K, p-Akt and Akt antibodies were obtained from Cell Signal Technology, Inc. (Danvers, MA, USA), while anti-Bax- ${ }_{\mathrm{L}}$, PSD95 and GAPDH antibodies were obtained from BD Transduction Laboratories (Breda, The Netherlands). Subsequently, the membranes incubated with primary antibodies were washed and incubated with horseradish peroxidase-conjugated secondary antibodies (anti-mouse: 1:1,000, catalog no. 61-6000; anti-Rabbit:
1:1,000, catalog no. A21253, Invitrogen; Thermo Fisher Scientific, Inc.) for $1 \mathrm{~h}$. The polypeptide bands were visualized using a Tanon-5200 Chemiluminescent Imaging System (Tanon Science \& Technology Co., Ltd. Shanghai, China). Images were quantified using ImageJ software version 1.44 (National Institutes of Health, Bethesda, MD, USA).

Statistical analysis. Statistical analysis was performed using SPSS software (version 19.0; IBM Corp., Armonk, NY, USA). All data are expressed as the mean \pm standard deviation. Differences between two groups were assessed by Tukey's test, while those among more groups were assessed using analysis of variance. $\mathrm{P}<0.05$ was considered to indicate differences that were statistically significant.

\section{Results}

Expression of miR-383 in the hippocampus following propofol anesthesia. Rats were divided into the untreated control, propofol anesthesia (propofol group, $n=12$ ), and miR-383 mimic or scramble treatment prior to propofol anesthesia (mimic/scramble + propofol) groups. Initially, the expression of miR-383 in the hippocampus of animals in the control or three propofol groups was determined using RT-qPCR. The results demonstrated that the expression of miR-383 in the propofol group was significantly reduced by propofol administration in comparison with the control group ( $\mathrm{P}<0.05$; Fig. 1A). However, miR-383 expression in the miR-383 mimic + propofol group was elevated following injection of the lentivirus expression vector, when compared with the expression in the control, propofol model and scramble + propofol groups $(\mathrm{P}<0.05$; Fig. 1A). Furthermore, no significant difference was observed in miR-383 expression between the miR-383 mimic + propofol and the miR-383 scramble + propofol groups. These data suggested that the miR-383-expressing lentivirus vector was successfully constructed.

miR-383 protects against reduced animal learning and memory ability induced by propofol anesthesia. MWM tests were performed to determine the influence of propofol, as well as the protective effect of miR-383, on animal learning and memory ability. Data from MWM test revealed that propofol administration markedly lengthened the time that rats required to find the hidden platform compared with the control group $(\mathrm{P}<0.05$; Fig. 1B). Furthermore, the animals treated with miR-383-expressing vector presented shorter latency time in comparison with the propofol model or scramble + propofol groups $(\mathrm{P}<0.05$; Fig. 1B). However, there were no significant differences in the swimming speed among the various groups $(\mathrm{P}>0.05$; Fig. $1 \mathrm{C})$. These results revealed that the miR-383-expressing lentivirus vector treatment protected against propofol-induced damage in animal learning and memory ability.

miR-383 inhibits propofol anesthesia-induced neuron apoptosis. At 7 days after the anesthesia, $4-\mu \mathrm{m}$ paraffin-embedded hippocampal sections were prepared for TUNEL assay. The results from TUNEL assay revealed that propofol anesthesia significantly increased neuron apoptosis, in comparison with the control group $(\mathrm{P}<0.05$; Fig. 2$)$. As 


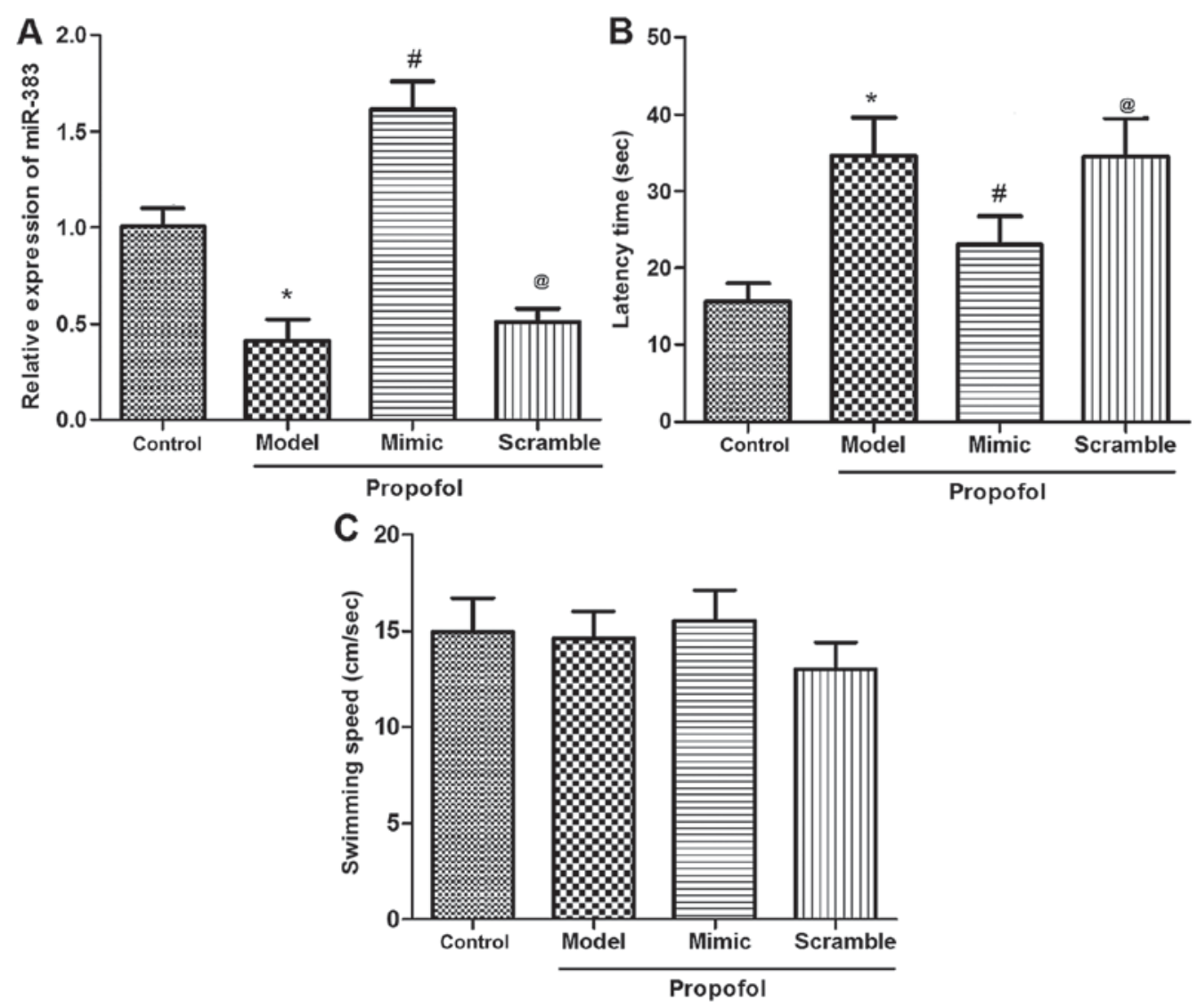

Figure 1. miR-383 expression in the hippocampus and its effect on animal learning and memory ability. (A) miR-383 expression level was detected using reverse transcription-quantitative polymerase chain reaction. (B) Latency time and (C) swimming speed were performed to examine the learning and memory abilities using the Morris water maze test. The latency was positively correlated with rat spatial memory. Lentiviruses containing miR-383 mimic or scramble vectors were injected to the hippocampus on the right cortex just above the dorsal hippocampus. ${ }^{*} \mathrm{P}<0.05$ vs. control; ${ }^{*} \mathrm{P}<0.05$ vs. propofol model group; ${ }^{\circledR} \mathrm{P}<0.05$ vs. mimic + propofol group. miR, microRNA.

expected, there was a reduction in neuron apoptosis in rats treated with miR-383-expressing lentivirus vector, when comparing with the control rats $(\mathrm{P}<0.05 ;$ Fig. 2). No significant difference was observed in the neuron apoptosis rate between the propofol model and the miR-383 scramble + propofol groups. These findings suggest that miR-383 may have inhibited the propofol anesthesia-induced damage on animal learning and memory ability by inhibiting neuron apoptosis.

miR-383 inhibits propofol anesthesia-induced dysregulation of apoptotic proteins. Based on the TUNEL assay, it was speculated that both propofol and miR-383 affected the expression of apoptosis-associated proteins. Thus, the present study detected the expression of two key apoptotic factors, Bcl-2 and Bax $(28,29)$. The mRNA and protein expression levels of Bcl-2 were significantly reduced, while those of Bax were significantly upregulated, by propofol anesthesia compared with the levels in the control group $(\mathrm{P}<0.05$; Fig. 3$)$. Furthermore, the propofol-induced dysregulation of $\mathrm{Bcl}-2$ and Bax was evidently inhibited in the miR-383-treated group, compared with the propofol anethesia model group $(\mathrm{P}<0.05$; Fig. 3). However, no significant difference was observed in the expression levels of Bcl-2 and Bax between the propofol model and miR-383 scramble + propofol groups. These data suggested that miR-38 mimic inhibited neuron apoptosis via regulating Bcl-2 and Bax expression.
Effects of propofol and miR-383 expression on neurodevelopment-associated protein expression. As previously reported, PSD95 and CREB are associated with and serve crucial roles in the development of hippocampal neurons $(30,31)$. Thus, the present study detected the effect of propofol administration, as well as of miR-383-expressing vectors, on the expression levels of PSD95 and CREB. Data from RT-qPCR and western blot analysis revealed that the mRNA and protein expression levels, respectively, of PSD95 and CREB were significantly inhibited by propofol administration $(\mathrm{P}<0.05 ;$ Fig. 4). In the mimic-treated group, however, the expression levels of PSD95 and CREB in the rat hippocampus were significantly upregulated compared with those of rats in the propofol, scramble + propofol and control groups $(\mathrm{P}<0.05$; Fig. 4). This demonstrated that miR-383 mimic administration contributed to the promotion of the propofol-damaged development of hippocampal neurons.

Effects of propofol and miR-383 expression on PI3K/Akt signaling pathway-associated protein expression. Since the PI3K-Akt signaling pathway is essential for cell apoptosis $(32,33)$, the expression and activated status of this pathway in animals were detected in the current study in order to examine the action mechanism of propofol and miR-383 expression on neuron apoptosis. The expression of p-PI3K and p-Akt mRNA and protein levels in the propofol-anesthetized 

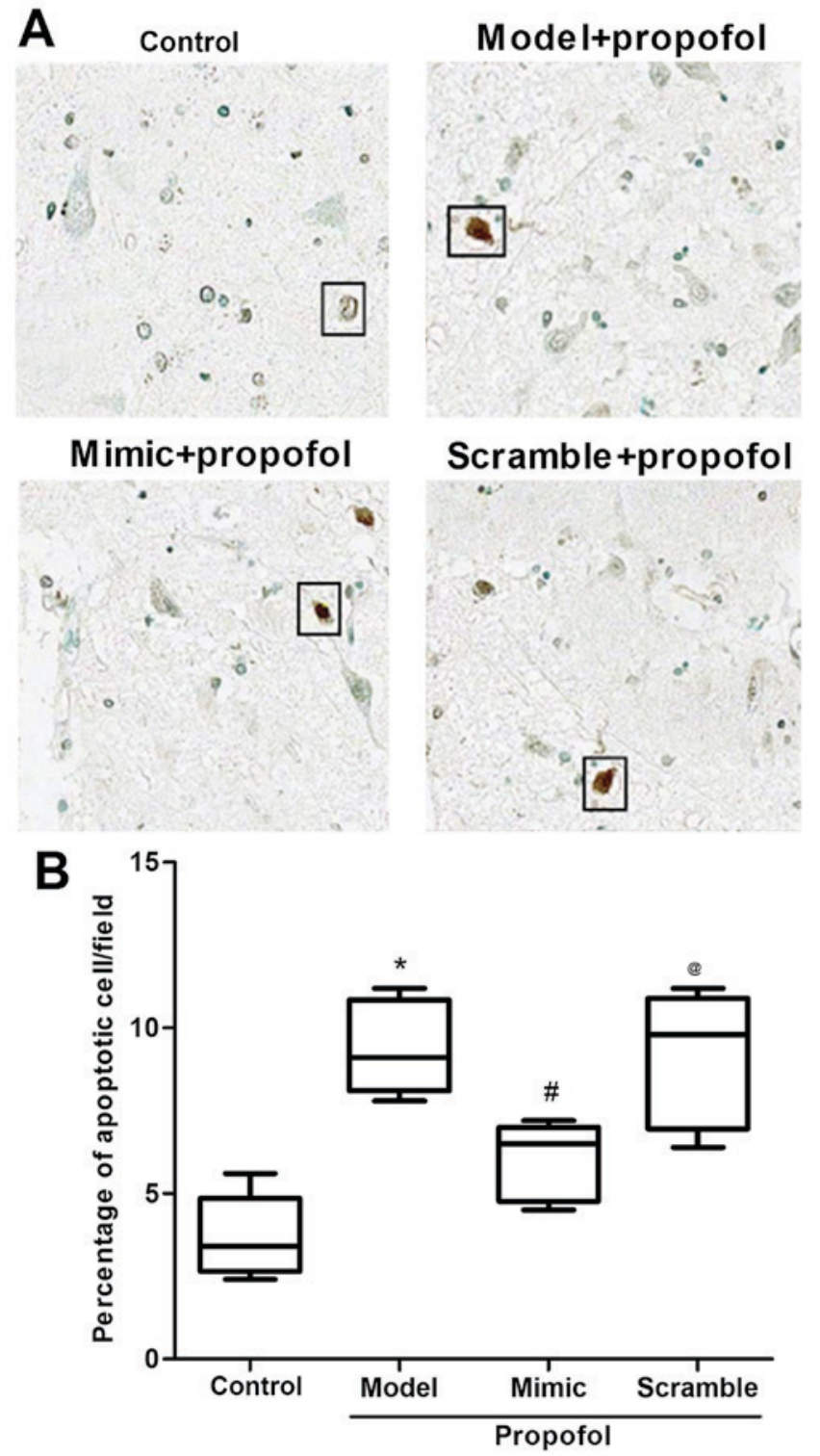

Figure 2. miR-383 inhibits propofol-induced hippocampus neuron apoptosis. (A) Neuron apoptosis images (Magnification, $\mathrm{x} 400$ ) and (B) percentage of apoptotic cells was examined using terminal deoxynucleotidyl transferase dUTP nick end labeling TUNEL assay. Cells in five arbitrarily selected fields were counted to determine the percentage of TUNEL positive cells. ${ }^{*} \mathrm{P}<0.05$ vs. control; ${ }^{\#} \mathrm{P}<0.05$ vs. propofol model group; ${ }^{\circledR} \mathrm{P}<0.05$ vs. mimic + propofol group. miR, microRNA.

group were significantly reduced in comparison with those in the control group ( $\mathrm{P}<0.05$; Fig. 5). By contrast, the miR-383-expressing vector triggered the expression of $\mathrm{p}-\mathrm{PI} 3 \mathrm{~K}$ and p-Akt, in comparison with the propofol group $(\mathrm{P}<0.05$; Fig. 5). There were no notable differences in the expression of total PI3K and Akt among the various groups, or in the expression of $\mathrm{p}-\mathrm{PI} 3 \mathrm{~K}$ and $\mathrm{p}$-Akt between rats treated with propofol and with miR-383 or scramble + propofol (Fig. 5B). These data demonstrated that propofol and miR-383 may regulate the activation of PI3K-Akt signaling pathway.

\section{Discussion}

Anesthesia-induced cognitive impairment is a recognized clinical phenomenon and possibly the most frequent type of
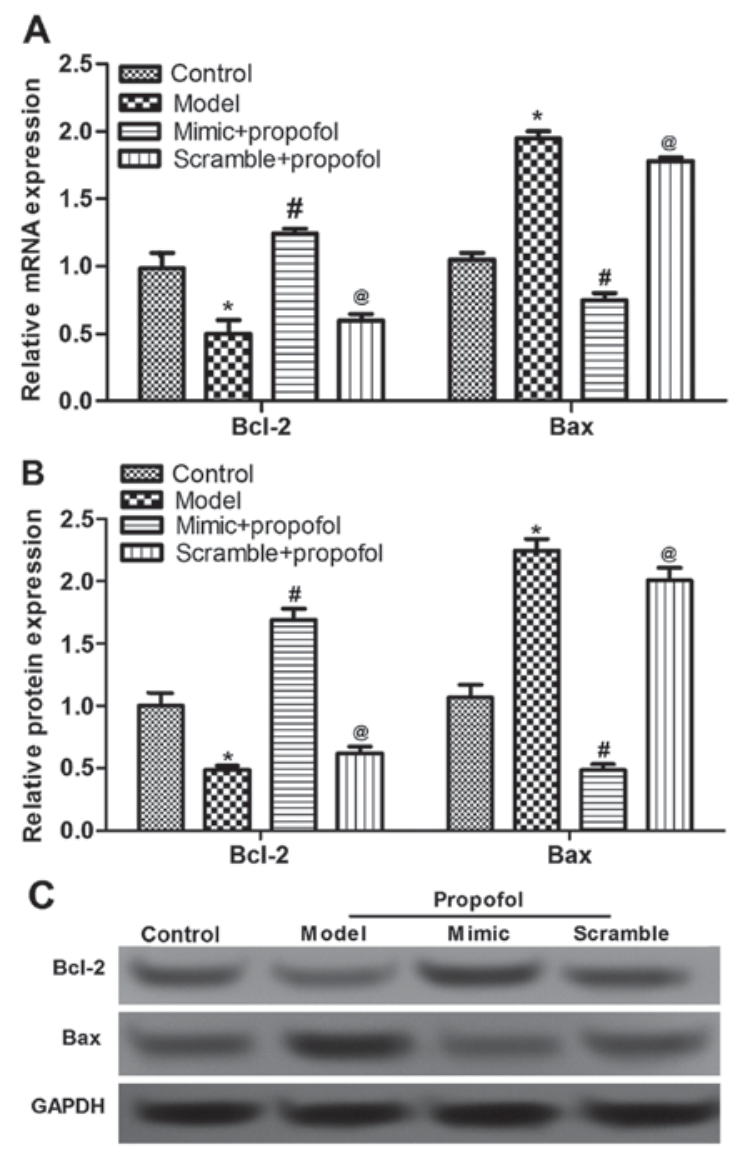

Figure 3. miR-383 inhibits propofol-dysregulated expression of Bcl-2 and Bax in hippocampus tissues. (A) Relative mRNA expression of these apoptotic proteins in the hippocampus were detected using reverse transcription-quantitative polymerase chain reaction, while the relative protein expression was demonstrated in the (B) quantified results and (C) blots of western blot analysis. ${ }^{*} \mathrm{P}<0.05$ vs. control; ${ }^{*} \mathrm{P}<0.05$ vs. propofol model group; ${ }^{\circledR} \mathrm{P}<0.05$ vs. mimic + propofol group. miR, microRNA; Bcl-2, B-cell lymphoma-2; Bax, Bcl-2-associated X protein.

postoperative cognitive impairment $(3,4)$. miRNAs had been reported to be associated with cognitive ability $(34,35)$. The present study aimed to investigate the effect of miR-383 expression on propofol-induced learning and memory impairment. The current results revealed that propofol anesthesia induced the downregulation of miR-383 expression. Further experiments demonstrated that miR-383-expressing vector treatment was able to repair the propofol anesthesia-induced cognitive impairment by inhibiting neuron cell apoptosis and by modulating the expression of apoptotic proteins and other factors associated with neuronal development, as well as by regulating the apoptosis signaling pathway.

Previous studies have demonstrated that anesthesia-induced cognitive impairment, including isoflurane, sevoflurane or propofol exposure, and the associated neuron apoptosis are recognized clinical phenomena $(36,37)$. Additionally, studies focusing on the comparison between propofol and other clinical anesthetics have observed that propofol has a controversial or dual effect on cognitive impairment (37-39). Propofol may induce cognitive impairment with a higher severity in comparison with sevoflurane administration (39). However, propofol may protect against or reduce cognitive impairment resulting from surgery or electroconvulsive therapy $(38,40)$. 

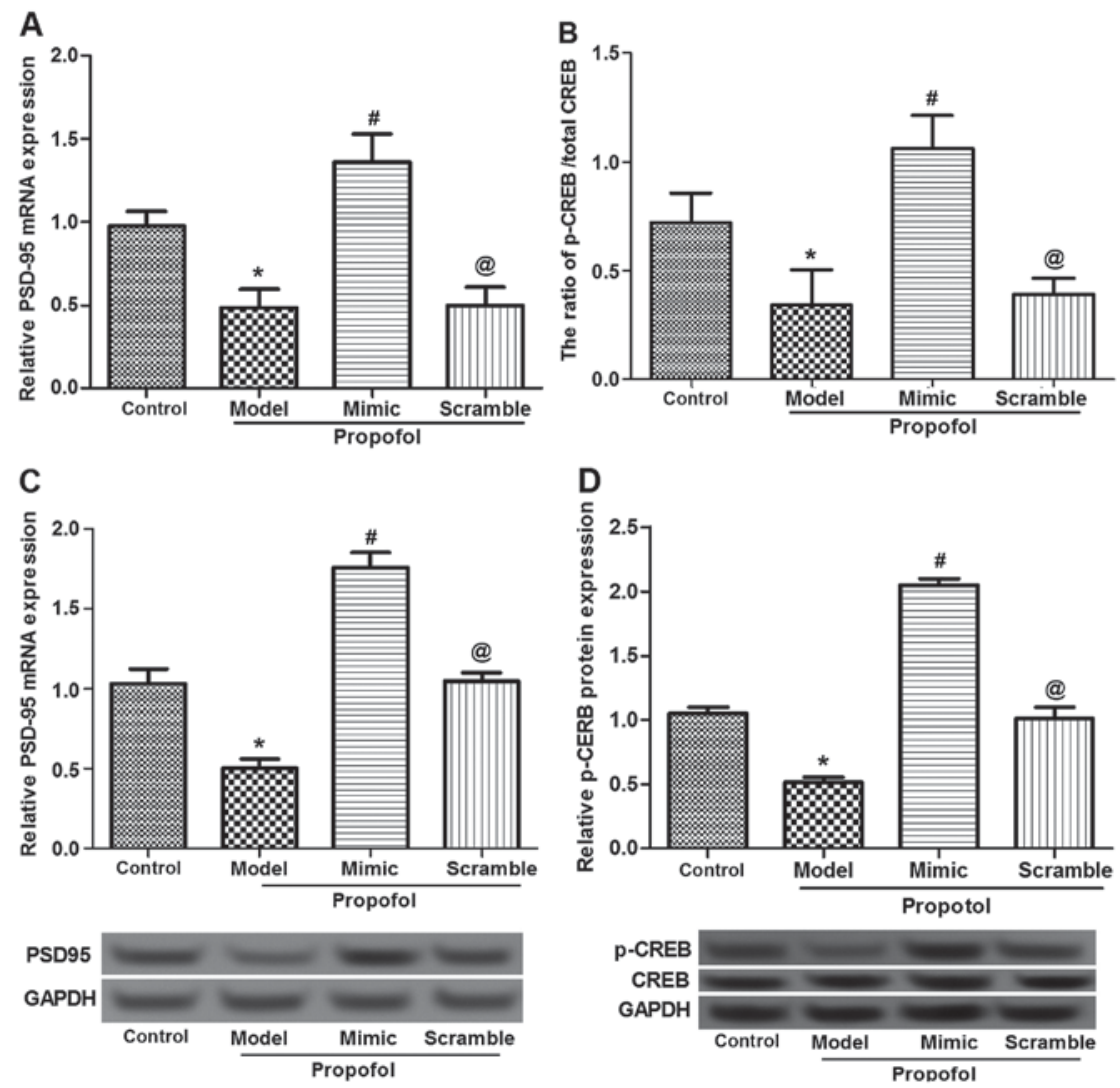

Figure 4. miR-383 expression promotes hippocampal neuron development. (A) PSD95 and (B) CREB mRNA expression levels were detected by reverse transcription-quantitative polymerase chain reaction. (C) PSD95 and (D) CREB protein expression levels were analyzed using western blot analysis. ${ }^{*} \mathrm{P}<0.05$ vs. control; " $\mathrm{P}<0.05$ vs. propofol model group; ${ }^{\circledR} \mathrm{P}<0.05$ vs. mimic + propofol group. miR, microRNA; PSD95, postsynaptic density protein 95 ; CREB, cAMP-response element binding protein.

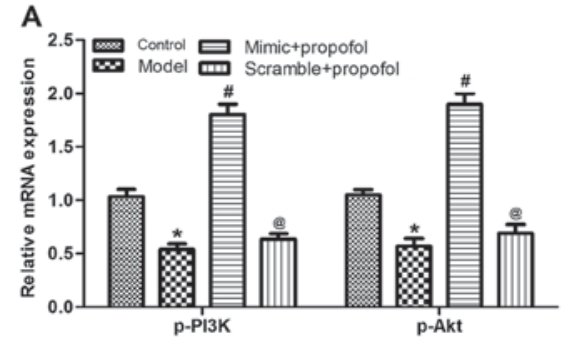

B

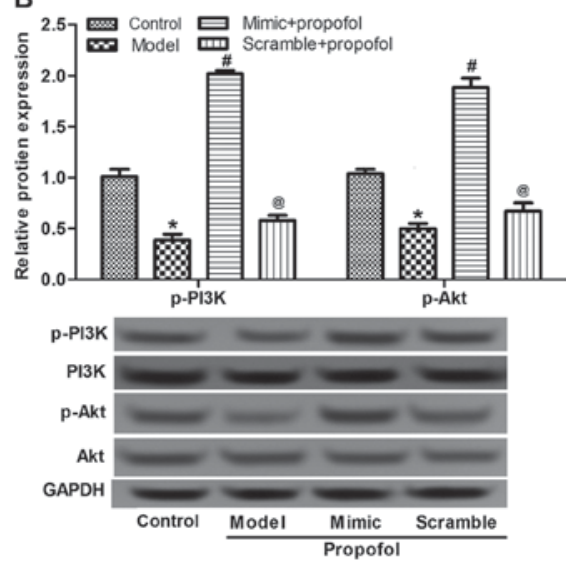

Figure 5. miR-383 expression triggers the activation of PI3K/Akt signaling pathway. The expression levels of the PI3K/Akt signaling pathway-associated proteins, $\mathrm{PI} 3 \mathrm{~K}$ and $\mathrm{Akt}$, were detected by (A) reverse transcription-quantitative polymerase chain reaction and (B) western blot analysis. " $\mathrm{P}<0.05$ vs. control; ${ }^{\text {"}} \mathrm{P}<0.05$ vs. propofol model group; ${ }^{\circledR} \mathrm{P}<0.05$ vs. mimic +propofol group. miR, microRNA; PI3K, phosphoinositide 3-kinase; Akt, protein kinase B.
In the present study, a rat model with cognitive impairment was successfully established using propofol administration. The increment in the apoptotic rate of hippocampal neurons (Fig. 2), alterations in apoptosis-associated protein expression (Figs. 3 and 4), as well as inhibition of the PI3K/Akt signaling pathway activation following propofol treatment (Fig. 5), demonstrated that propofol administration resulted in cognitive impairment in rats.

miR-383 is a disease-associated miRNA, and its downregulation has been identified in patients with various types of cancer, infertility and other diseases $(23,24)$. Ectopic expression of miR-383 has been demonstrated to be associate with cell growth, apoptosis and expression of apoptosis-associated proteins (22). In the present study, miR-383 mimic-expressing lentivirus vectors were successfully constructed and employed as a treatment for propofol-induced cognitive impairment. Administration of miR-383 mimics significantly upregulated the reduced expression levels of miR-383, neuron development factors and PI3K/Akt signaling pathway factors, $\mathrm{p}-\mathrm{PI} 3 \mathrm{~K}$ and p-Akt, that resulted from propofol administration. In addition, miR-383 mimics inhibited the propofol-altered expression of apoptosis-associated proteins and apoptotic neuron cells. These observations revealed that miR-383 repaired the propofol-induced cognitive impairment in the rat model, and that it may be a novel target for exploring effective therapeutic strategies for propofol anesthesia-induced cognitive impairment.

Cell apoptosis is a complicate process involved in numerous signaling pathways, as well as stimulation, genetic and 
non-genetic factors (41-44). Cognitive impairments resulting from propofol or other anesthetics have been demonstrated to involve hippocampal neuron apoptosis and regulation of apoptosis-associated proteins and signaling pathways $(37,45,46)$. For instance, Bcl-2 and Bax are important factors for cell apoptosis, which is benefited by overexpression of Bax and inhibition of $\mathrm{Bcl}-2$, or upregulation of the $\mathrm{Bax} / \mathrm{Bcl}-2$ ratio $(16,17,47,48)$. In addition, Bcl-2 expression may be mediated by p-CREB or CREB-dependent Bcl-2 signaling, which further contributes to cell apoptosis $(49,50)$. Furthermore, CREB is required for PSD95 disruption (31), and the transcription of PSD95 may be directly activated CREB (51). In the current study, it was determined that the propofol anesthesia upregulated $\mathrm{Bax} / \mathrm{Bcl}-2$ ratio, but this was then reduced by miR-383 mimic transfection via inhibiting Bax and inducing Bcl-2 (Fig. 3). Additionally, propofol anesthesia-inhibited PSD95 and p-CREB expression levels were upregulated by miR-383 mimic administration (Fig. 4). These findings demonstrated that the apoptotic factors Bax and Bcl-2, as well as PSD95 and p-CREB, responded to the abnormal expression of miR-383.

$\mathrm{PI} 3 \mathrm{~K} / \mathrm{Akt}$ signaling pathway is a key pathway for cell apoptosis $(16,17)$. As reported, inhibition of the PI3K/Akt signaling pathway contributed to cancer cell apoptosis (18). Yoshii and Constantine-Paton revealed that the inhibition of MAPK/ERK signaling pathway disrupted PSD95 expression, and that PI3K is critical for the PSD95 disruption (51). However, there was no evidence demonstrating the effect of miR-383 mimic on PI3K/Akt signaling pathway activation in propofol anesthetized animals with cognitive impairment. Since miR-383 dysregulation and PI3K/Akt signaling pathway participate in cell apoptosis $(16,17,23,24)$, the present study speculated that miR-383 mimics may be able to regulate the expression levels of $\mathrm{p}-\mathrm{PI} 3 \mathrm{~K}$ and $\mathrm{p}-\mathrm{Akt}$, and the activation status factors of PI3K/Akt signaling pathway. As expected, propofol administration significantly downregulated the expression levels of p-PI3K and p-Akt proteins (Fig. 5), as well as those of miR-383, Bax/Bcl-2, PSD95 and p-CREB. However, miR-383 mimic evidently upregulated the expression of all these factors, revealing that miR-383 regulated the expression or activation of PI3K/Akt signaling pathway, thus modulating cell apoptosis.

In conclusion, in the present study, a cognitive impairment rat model was established using propofol anesthesia. Propofol induced downregulation of miR-383, lengthened latency time, apoptosis of hippocampal neurons, upregulation of $\mathrm{Bax} / \mathrm{Bcl}-2$, inhibition of PSD95 and p-CREB expression levels, and inactivation of PI3K/Akt signaling pathway. However, treatment with miR-383 mimic-expressing lentivirus vector significantly improved the propofol effects, demonstrating the protective effect of miR-383 on propofol anesthesia-induced cognitive impairment in the rat model. The current study also suggested the miR-383 mimics may be used as a novel target for the development of a therapeutic strategy for propofol anesthesia-induced cognitive impairment.

\section{References}

1. Parrott MD, Winocur G, Bazinet RP, Ma DW and Greenwood CE: Whole-food diet worsened cognitive dysfunction in an Alzheimer's disease mouse model. Neurobiol Aging 36: 90-99, 2015 .
2. Ng CL, Cheng OY, Kwan SC, Ho WL, Cheng KY, Chung SK, Lam KSL, Xu A and Chan KH: Adiponectin deficiency induced cognitive dysfunction and Alzheimer's disease pathogenesis in mice through the disruption of insulin sensitivity and inactivated AMPK signaling. In: Proceedings of the 67th Annual Meeting of the American Academy of Neurology (AAN 2015). AAN, Washington, DC, 2015.

3. Rundshagen I: Postoperative cognitive dysfunction. Dtsch Arztebl Int 111: 119-125, 2014.

4. Grape S, Ravussin P, Rossi A, Kern C and Steiner L: Postoperative cognitive dysfunction. Trends Anaesthesia Crit Care 2: 98-103, 2012.

5. Tabaka P, Goodam S, Sommer BR, Maloney W, Huddleston J and Lemmens HJ: The effect of desdlurane versus propofol anesthesia on postoperative delirium in elderly obese patients undergoing total knee replacement: A randomized, controlled, double-blinded clinical trial. J Clin Anethesia 39: 17-22, 2017.

6. Vogelstein B and Kinzler KW: Cancer genes and the pathways they control. Nat Med 10: 789-799, 2004.

7. Wang Y, Wu C, Han B, Xu F, Mao M, Guo X and Wang J: Dexmedetomidine attenuates repeated propofol exposure-induced hippocampal apoptosis, PI3K/Akt/Gsk-3b siganling disruption, and juvenile cognitive deficits in neonatal rats. Mol Med Rep 14: 769-775, 2016

8. Shaltiel G, Hanan M, Wolf Y, Barbash S, Kovalev E, Shoham S and Soreq H: Hippocampal microRNA-132 mediates stress-inducible cognitive deficits through its acetylcholinesterase target. Brain Struct Funct 218: 59-72, 2013.

9. Green M, Cairns M, Wu J, Dragovic M, Jablensky A, Tooney PA, Scott RJ and Carr VJ; Australian Schizophrenia Research Bank: Genome-wide supported variant MIR137 and severe negative symptoms predict membership of an impaired cognitive subtype of schizophrenia. Mol Psychiatry 18: 774-780, 2013.

10. Takeda K, Kermani P, Anastasia A, Obinata Y, Hempstead BL and Kurihara $\mathrm{H}$ : BDNF protects human vascular endothelial cells from TNF $\alpha$-induced apoptosis. Biochem Cell Biol 91: 341-349, 2013.

11. Dong J, Wang Y, Wang Y, Wei W, Min H, Song B, Xi Q, Teng $\mathrm{W}$ and Chen $\mathrm{J}$ : Iodine deficiency increases apoptosis and decreases synaptotagmin-1 and PSD-95 in rat hippocampus. Nutr Neurosci 16: 135-141, 2013.

12. Liu R, Xing L, Kong D, Jiang J, Shang L and Hao W: Bisphenol A inhibits proliferation and induces apoptosis in micromass cultures of rat embryonic midbrain cells through the JNK, CREB and p53 signaling pathways. Food Chemical Toxicol 52: 76-82, 2013.

13. Zuo H, Lin T, Wang D, Peng R, Wang S, Gao Y, Xu X, Zhao L, Wang $\mathrm{S}$ and $\mathrm{Su} \mathrm{Z}$ : RKIP regulates neural cell apoptosis induced by exposure to microwave radiation partly through the MEK/ ERK/CREB pathway. Mol Neurobiol 51: 1520-1529, 2015.

14. Welch C, Chen Y and Stallings R: MicroRNA-34a functions as a potential tumor suppressor by inducing apoptosis in neuroblastoma cells. Oncogene 26: 5017-5022, 2007.

15. Zhao L, Gu H, Chang J, Wu J, Wang D, Chen S, Yang X and Qian B: MicroRNA-383 regulates the apoptosis of tumor cells through targeting Gadd45g. PLoS One 9: e110472, 2014.

16. Liu G, Wang T, Wang T, Song J and Zhou Z: Effects of apoptosis-related proteins caspase-3, Bax and Bcl-2 on cerebral ischemia rats. Biomed Rep 1: 861-867, 2013.

17. Liang K, Ye Y, Wang Y, Zhang J and Li C: Formononetin mediates neuroprotection against cerebral ischemia/reperfusion in rats via downregulation of the $\mathrm{Bax} / \mathrm{Bcl}-2$ ratio and upregulation PI3K/Akt signaling pathway. J Neurol Sci 344: 100-104, 2014.

18. Malla R, Gopinath S, Alapati K, Gondi CS, Gujrati M, Dinh DH, Mohanam S and Rao JS: Correction: Downregulation of uPAR and cathepsin $\mathrm{B}$ induces apoptosis via regulation of $\mathrm{Bcl}-2$ and $\mathrm{Bax}$ and inhibition of the PI3K/Akt pathway in gliomas. PLoS One 9: 2014.

19. Yao D, Zhang WR, He X, Wang JH, Jiang KW and Zhao ZY: The expression of PI3K/Akt signaling pathway and PTEN in hippocampus of the brain and the correlation with cognitive impairment after neonatal hypoxic ischemic brain damage in rats. Int J Clin Exp Med 9: 9044-9053, 2016.

20. Shu Y, Zhang H, Kang T, Zhang JJ, Yang Y, Liu H and Zhang L: PI3K/Akt signal pathway involved in the cognitive impairment caused by chronic cerebral hypoperfusion in rats. PLoS One 8: e81901, 2013.

21. Wang Y, Wu C, Han B, Xu F, Mao M, Guo X and Wang J: Dexmedetomidine attenuates repeated propofol exposure-induced hippocampal apoptosis, PI3K/Akt/Gsk-3 $\beta$ signaling disruption, and juvenile cognitive deficits in neonatal rats. Mol Med Rep 14: 769-775, 2016. 
22. Li KK, Pang JC, Lau KM, Zhou L, Mao Y, Wang Y, Poon WS and Ng HK: MiR-383 is downregulated in medulloblastoma and targets peroxiredoxin 3 (PRDX3). Brain Pathol 23: 413-425, 2013.

23. Lian J, Tian H, Liu L, Zhang XS, Li WQ, Deng YM, Yao GD, Yin MM and Sun F: Downregulation of microRNA-383 is associated with male infertility and promotes testicular embryonal carcinoma cell proliferation by targeting IRF1. Cell Death Dis 1: e94, 2010.

24. He Z, Cen D, Luo X, Li D, Li P, Liang L and Meng Z: Downregulation of miR-383 promotes glioma cell invasion by targeting insulin-like growth factor 1 receptor. Med Oncol 30: $557,2013$.

25. Cazzalini O, Scovassi AI, Savio M, Stivala LA and Prosperi E: Multiple roles of the cell cycle inhibitor p21(CDKN1A) in the DNA damage response. Mutat Res 704: 12-20, 2010.

26. Chakraborty C, Doss CG,Bandyopadhyay $S$ and Agoramoorthy G: Influence of miRNA in insulin signaling pathway and insulin resistance: Micro-molecules with a major role in type-2 diabetes. Wiley Interdiscip Rev RNA 5: 697-712, 2014.

27. Livak KJ and Schmittgen TD: Analysis of relative gene expression data using real-time quantitative PCR and the 2(-Delta Delta C(T)) method. Method 25: 402-408, 2001.

28. Smith CC, Guévremont D, Williams JM and Napper RM: Apoptotic cell death and temporal expression of apoptotic proteins $\mathrm{Bcl}-2$ and $\mathrm{Bax}$ in the hippocampus, following binge ethanol in the neonatal rat model. Alcohol Clin Exp Res 39: 36-44, 2015.

29. Cao J, Chen J, Wang J, Jia R, Xue W, Luo Y and Gan X: Effects of fluoride on liver apoptosis and Bcl-2, Bax protein expression in freshwater teleost, Cyprinus carpio. Chemosphere 91: 1203-1212, 2013.

30. Bustos FJ, Varela-Nallar L, Campos M, Henriquez B, Phillips M, Opazo C, Aguayo LG, Montecino M, Constantine-Paton M, Inestrosa NC and van Zundert B: PSD95 suppresses dendritic arbor development in mature hippocampal neurons by occluding the clustering of NR2B-NMDA receptors. PLoS One 9: e94037, 2014.

31. Bell KF, Bent RJ, Meese-Tamuri S, Ali A, Forder JP and Aarts MM: Calmodulin Kinase IV-dependent CREB activation is required for neuroprotection via NMDA receptor-PSD95 disruption. J Neurochem 126: 274-287, 2013.

32. Chen K, Li G, Geng F, Zhang Z, Li J, Yang M, Dong L and Gao F: Berberine reduces ischemia/reperfusion-induced myocardial apoptosis via activating AMPK and PI3K-Akt signaling in diabetic rats. Apoptosis 19: 946-957, 2014.

33. Roy R, Singh SK, Chauhan LK, Das M, Tripathi A and Dwivedi PD: Zinc oxide nanoparticles induce apoptosis by enhancement of autophagy via PI3K/Akt/mTOR inhibition. Toxicol Lett 227: 29-40, 2014.

34. Luo T, Yin S, Shi R, Xu C, Wang Y, Cai J, Yue Y and Wu A miRNA expression profile and involvement of Let-7d-APP in aged rats with isoflurane-induced learning and memory impairment. PLoS One 10: e0119336, 2015.

35. Liu W, Liu C, Zhu J, Shu P, Yin B, Gong Y, Qiang B, Yuan J and Peng X: MicroRNA-16 targets amyloid precursor protein to potentially modulate Alzheimer's-associated pathogenesis in SAMP8 mice. Neurobiol Aging 33: 522-534, 2012.

36. Liu Y, Wang XJ, Wang N, Cui CL and Wu LZ: Electroacupuncture Ameliorates propofol-induced cognitive impairment via an opioid receptor-independent mechanism. Am J Chin Med 44: 705-719, 2016.

37. Yang B, Liang G, Khojasteh S, Wu Z, Yang W, Joseph D and Wei $\mathrm{H}$ : Comparison of neurodegeneration and cognitive impairment in neonatal mice exposed to propofol or isoflurane. PLoS One 9: e99171, 2014.
38. Luo J, Min S, Wei K, Li P, Dong J and Liu YF: Propofol protects against impairment of learning-memory and imbalance of hippocampal Glu/GABA induced by electroconvulsive shock in depressed rats. J Anesth 25: 657-665, 2011.

39. Schoen J,Husemann L, Tiemeyer C, Lueloh A, Sedemund-Adib B, Berger KU, Hueppe M and Heringlake M: Cognitive function after sevoflurane-vs propofol-based anaesthesia for on-pump cardiac surgery: A randomized controlled trial. Br J Anaesth 106: 840-850, 2011.

40. Butterfield NN, Graf P, Macleod BA, Ries CR and Zis AP: Propofol reduces cognitive impairment after electroconvulsive therapy. J ECT 20: 3-9, 2004

41. Pan HC, Jiang Q, Yu Y, Mei JP, Cui YK and Zhao WJ: Quercetin promotes cell apoptosis and inhibits the expression of MMP-9 and fibronectin via the AKT and ERK signalling pathways in human glioma cells. Neurochem Int 80: 60-71, 2015.

42. Romanov V, Whyard TC, Waltzer WC, Grollman AP and Rosenquist T: Aristolochic acid-induced apoptosis and G2 cell cycle arrest depends on ROS generation and MAP kinases activation. Arch Toxicol 89: 47-56, 2015.

43. Choe JY, Park KY and Kim SK: Oxidative stress by monosodium urate crystals promotes renal cell apoptosis through mitochondrial caspase-dependent pathway in human embryonic kidney 293 cells: Mechanism for urate-induced nephropathy. Apoptosis 20: 38-49, 2015.

44. Zhang Y, Han L, Qi W, Cheng D, Ma X, Hou L, Cao X and Wang C: Eicosapentaenoic acid (EPA) induced apoptosis in HepG2 cells through ROS-Ca (2+)-JNK mitochondrial pathways. Biochem Biophys Res Commun 456: 926-932, 2015.

45. Zhang D, Zhou XH, Zhang J, Zhou YX, Ying J, Wu GQ and Qian JH: Propofol promotes cell apoptosis via inhibiting HOTAIR mediated mTOR pathway in cervical cancer. Biochem Biophys Res Commun 468: 561-567, 2015.

46. Li XM, Zhou MT, Wang XM, Ji MH, Zhou ZQ and Yang JJ: Resveratrol pretreatment attenuates the isoflurane-induced cognitive impairment through its anti-inflammation and-apoptosis actions in aged mice. J Mol Neurosci 52: 286-293, 2014.

47. Yap JL, Cao X, Vanommeslaeghe K, Jung KY, Peddaboina C, Wilder PT, Nan A, MacKerell AD Jr, Smythe WR and Fletcher S: Relaxation of the rigid backbone of an oligoamide-foldamer-based $\alpha$-helix mimetic: Identification of potent Bcl-x L inhibitors. Org Biomol Chem 10: 2928-2933, 2012.

48. Yue J, Ben Messaoud N and López JM: Hyperosmotic shock engages two positivefeedback loops through Caspase-3-dependent Proteolysis of JNK1-2 and Bid. J Biol Chem 290: 30375-30389, 2015.

49. Das S, Cordis GA, Maulik N and Das DK: Pharmacological preconditioning with resveratrol: Role of CREB-dependent Bcl-2 signaling via adenosine A3 receptor activation. Am J Physiol Heart Circ Physiol 288: H328-H335, 2005.

50. Fujii M, Sherchan P, Soejima Y,Hasegawa Y, Flores J, Doycheva D and Zhang JH: Cannabinoid receptor type 2 agonist attenuates apoptosis by activation of phosphorylated CREB-Bcl-2 pathway after subarachnoid hemorrhage in rats. Exp Neurol 261: 396-403, 2014.

51. Yoshii A and Constantine-Paton M: Postsynaptic localization of PSD-95 is regulated by all three pathways downstream of TrkB signaling. Front Synaptic Neurosci 6: 6, 2014

This work is licensed under a Creative Commons Attribution-NonCommercial-NoDerivatives 4.0 International (CC BY-NC-ND 4.0) License. 